\title{
Extremal values of Dirichlet $L$-functions in the half-plane of absolute convergence
}

\author{
par JÖRN STEUDING
}

RÉSUMÉ. On démontre que, pour tout $\theta$ réel, il existe une infinité de $s=\sigma+i t$ avec $\sigma \rightarrow 1+$ et $t \rightarrow+\infty$ tel que

$$
\operatorname{Re}\{\exp (i \theta) \log L(s, \chi)\} \geq \log \frac{\log \log \log t}{\log \log \log \log t}+O(1) .
$$

La démonstration est basée sur une version effective du théorème de Kronecker sur les approximations diophantiennes.

Abstract. We prove that for any real $\theta$ there are infinitely many values of $s=\sigma+i t$ with $\sigma \rightarrow 1+$ and $t \rightarrow+\infty$ such that

$$
\operatorname{Re}\{\exp (i \theta) \log L(s, \chi)\} \geq \log \frac{\log \log \log t}{\log \log \log \log t}+O(1) .
$$

The proof relies on an effective version of Kronecker's approximation theorem.

\section{Extremal values}

Extremal values of the Riemann zeta-function in the half-plane of absolute convergence were first studied by $\mathrm{H}$. Bohr and Landau [1]. Their results rely essentially on the diophantine approximation theorems of Dirichlet and Kronecker. Whereas everything easily extends to Dirichlet series with real coefficients of one sign (see [7], §9.32) the question of general Dirichlet series is more delicate. In this paper we shall establish quantitative results for Dirichlet $L$-functions.

Let $q$ be a positive integer and let $\chi$ be a Dirichlet character mod $q$. As usual, denote by $s=\sigma+i t$ with $\sigma, t \in \mathbb{R}, i^{2}=-1$, a complex variable. Then the Dirichlet $L$-function associated to the character $\chi$ is given by

$$
L(s, \chi)=\sum_{n=1}^{\infty} \frac{\chi(n)}{n^{s}}=\prod_{p}\left(1-\frac{\chi(p)}{p^{s}}\right)^{-1},
$$

where the product is taken over all primes $p$; the Dirichlet series, and so the Euler product, converge absolutely in the half-plane $\sigma>1$. Denote by

Manuscrit reçu le 9 juillet 2002. 
$\chi_{0}$ the principal character $\bmod q$, i.e., $\chi_{0}(n)=1$ for all $n$ coprime with $q$. Then

$$
L\left(s, \chi_{0}\right)=\zeta(s) \prod_{p \mid q}\left(1-\frac{1}{p^{s}}\right) .
$$

Thus we may interpret the well-known Riemann zeta-function $\zeta(s)$ as the Dirichlet $L$-function to the principal character $\chi_{0} \bmod 1$. Furthermore, it follows that $L\left(s, \chi_{0}\right)$ has a simple pole at $s=1$ with residue 1 . On the other side, any $L(s, \chi)$ with $\chi \neq \chi_{0}$ is regular at $s=1$ with $L(1, \chi) \neq 0$ (by Dirichlet's analytic class number-formula). Since $L(s, \chi)$ is non-vanishing in $\sigma>1$, we may define the logarithm (by choosing any one of the values of the logarithm). It is easily shown that for $\sigma>1$

$$
\log L(s, \chi)=\sum_{p} \sum_{k \geq 1} \frac{\chi(p)^{k}}{k p^{k s}}=\sum_{p} \frac{\chi(p)}{p^{s}}+O(1) .
$$

Obviously, $|\log L(s, \chi)| \leq L\left(\sigma, \chi_{0}\right)$ for $\sigma>1$. However

Theorem 1.1. For any $\epsilon>0$ and any real $\theta$ there exists a sequence of $s=\sigma+i t$ with $\sigma>1$ and $t \rightarrow+\infty$ such that

$$
R e\{\exp (i \theta) \log L(s, \chi)\} \geq(1-\epsilon) \log L\left(\sigma, \chi_{0}\right)+O(1) .
$$

In particular,

$$
\liminf _{\sigma>1, t \geq 1}|L(s, \chi)|=0 \quad \text { and } \quad \limsup _{\sigma>1, t \geq 1}|L(s, \chi)|=\infty .
$$

In spite of the non-vanishing of $L(s, \chi)$ the absolute value takes arbitrarily small values in the half-plane $\sigma>1$ !

The proof follows the ideas of H. Bohr and Landau [1] (resp. [8], §8.6) with which they obtained similar results for the Riemann zeta-function (answering a question of Hilbert). However, they argued with Dirichlet's homogeneous approximation theorem for growth estimates of $|\zeta(s)|$ and with Kronecker's inhomogeneous approximation theorem for its reciprocal. We will unify both approaches.

Proof. Using (2) we have for $x \geq 2$

(3) $\operatorname{Re}\{\exp (i \theta) \log L(s, \chi)\}$

$$
\geq \sum_{p \leq x} \frac{\chi_{0}(p)}{p^{\sigma}} \operatorname{Re}\left\{\exp (i \theta) \chi(p) p^{-i t}\right\}-\sum_{p>x} \frac{\chi_{0}(p)}{p^{\sigma}}+O(1) .
$$


Denote by $\varphi(q)$ the number of prime residue classes $\bmod q$. Since the values $\chi(p)$ are $\varphi(q)$-th roots of unity if $p$ does not divide $q$, and equal to zero otherwise, there exist integers $\lambda_{p}$ (uniquely determined $\bmod \varphi(q)$ ) with

$$
\chi(p)=\left\{\begin{array}{ccc}
\exp \left(2 \pi i \frac{\lambda_{p}}{\varphi(q)}\right) & \text { if } & p \not q \\
0 & \text { if } & p \mid q .
\end{array}\right.
$$

Hence,

$$
\operatorname{Re}\left\{\exp (i \theta) \chi(p) p^{-i t}\right\}=\cos \left(t \log p-2 \pi \frac{\lambda_{p}}{\varphi(q)}-\theta\right) .
$$

In view of the unique prime factorization of the integers the logarithms of the prime numbers are linearly independent. Thus, Kronecker's approximation theorem (see [8], §8.3, resp. Theorem 3.2 below) implies that for any given integer $\omega$ and any $x$ there exist a real number $\tau>0$ and integers $h_{p}$ such that

$$
\left|\frac{\tau}{2 \pi} \log p-\frac{\lambda_{p}}{\varphi(q)}-\frac{\theta}{2 \pi}-h_{p}\right|<\frac{1}{\omega} \quad \text { for all } \quad p \leq x .
$$

Obviously, with $\omega \rightarrow \infty$ we get infinitely many $\tau$ with this property. It follows that

$$
\cos \left(\tau \log p-2 \pi \frac{\lambda_{p}}{\phi(q)}-\theta\right) \geq \cos \left(\frac{2 \pi}{\omega}\right) \quad \text { for all } \quad p \leq x,
$$

provided that $\omega \geq 4$. Therefore, we deduce from (3)

$$
\operatorname{Re}\{\exp (i \theta) \log L(\sigma+i \tau, \chi)\} \geq \cos \left(\frac{2 \pi}{\omega}\right) \sum_{p \leq x} \frac{\chi_{0}(p)}{p^{\sigma}}-\sum_{p>x} \frac{\chi_{0}(p)}{p^{\sigma}}+O(1),
$$

resp.

(6) $\operatorname{Re}\{\exp (i \theta) \log L(\sigma+i \tau, \chi)\}$

$$
\geq \cos \left(\frac{2 \pi}{\omega}\right) \log L\left(\sigma, \chi_{0}\right)-2 \sum_{p>x} \frac{1}{p^{\sigma}}+O(1)
$$

in view of (2). Obviously, the appearing series converges. Thus, sending $\omega$ and $x$ to infinity gives the inequality of Theorem 1.1. By (1) we have

$$
\log L\left(\sigma, \chi_{0}\right)=\log \left(\frac{1}{\sigma-1}+O(1)\right)=\log \frac{1}{\sigma-1}+o(1)
$$

for $\sigma \rightarrow 1+$. Therefore, with $\theta=0$, resp. $\theta=\pi$, and $\sigma \rightarrow 1+$ the further assertions of the theorem follow.

The same method applies to other Dirichlet series as well. For example, one can show that the Lerch zeta-function is unbounded in the half-plane 
of absolute convergence:

$$
\limsup _{\sigma>1, t \geq 1} \sum_{n=0}^{\infty} \frac{\exp (2 \pi i \lambda n)}{(n+\alpha)^{s}}=+\infty
$$

if $\alpha>0$ is transcendental; note that in the case of transcendental $\alpha$ the Lerch zeta-function has zeros in $\sigma>1$ (see [3] and [4]).

In view of Theorem 1.1 we have to ask for quantitative estimates. Let $\pi(x)$ count the prime numbers $p \leq x$. By partial summation,

$$
\sum_{x<p \leq y} \frac{1}{p^{\sigma}}=\frac{\pi(y)}{y^{\sigma}}-\frac{\pi(x)}{x^{\sigma}}+\sigma \int_{x}^{y} \frac{\pi(u)}{u^{\sigma+1}} \mathrm{~d} u
$$

The prime number theorem implies for $x \geq 2$

$$
\sum_{x<p \leq y} \frac{1}{p^{\sigma}} \sim\left(\frac{y^{1-\sigma}}{\log y}-\frac{x^{1-\sigma}}{\log x}\right)+\sigma \int_{x}^{y} \frac{\mathrm{d} u}{u^{\sigma} \log u} .
$$

By the second mean-value theorem,

$$
\int_{x}^{y} \frac{\mathrm{d} u}{u^{\sigma} \log u} \mathrm{~d} u=\frac{1}{\log \xi} \int_{x}^{y} \frac{\mathrm{d} u}{u^{\sigma}}=\frac{x^{1-\sigma}-y^{1-\sigma}}{(\sigma-1) \log \xi}
$$

for some $\xi \in(x, y)$. Thus, substituting $\xi$ by $x$ and sending $y \rightarrow \infty$, we obtain the estimate

$$
\sum_{x<p} \frac{1}{p^{\sigma}} \leq(1+o(1)) \frac{x^{1-\sigma}}{(\sigma-1) \log x}
$$

as $x \rightarrow \infty$. This gives in (6)

(8) $\operatorname{Re}\{\exp (i \theta) \log L(\sigma+i \tau, \chi)\}$

$$
\geq \cos \left(\frac{2 \pi}{\omega}\right) \log L\left(\sigma, \chi_{0}\right)-(2+o(1)) \frac{x^{1-\sigma}}{(\sigma-1) \log x}+O(1) .
$$

Substituting (7) in formula (8) yields

$$
\begin{aligned}
\operatorname{Re}\{\exp (i \theta) & \log L(\sigma+i \tau, \chi)\} \\
& \geq\left(1+O\left(\omega^{-2}\right)\right) \log \frac{1}{\sigma-1}-(2+o(1)) \frac{x^{1-\sigma}}{(\sigma-1) \log x}+O(1) .
\end{aligned}
$$

Let

$$
x=\exp \left(\frac{1}{\sigma-1} \log \frac{1}{\sigma-1}\right),
$$

then $x$ tends to infinity as $\sigma \rightarrow 1+$. We obtain for $x$ sufficiently large

$$
\operatorname{Re}\{\exp (i \theta) \log L(\sigma+i \tau, \chi)\} \geq\left(1+O\left(\omega^{-2}\right)\right) \log \frac{\log x}{\log \log x}+O(1) .
$$

The question is how the quantities $\omega, x$ and $\tau$ depend on each other. 


\section{Effective approximation}

H. Bohr and Landau [2] (resp. [8], §8.8) proved the existence of a $\tau$ with $0 \leq \tau \leq \exp \left(N^{6}\right)$ such that

$$
\cos \left(\tau \log p_{\nu}\right)<-1+\frac{1}{N} \quad \text { for } \quad \nu=1, \ldots, N,
$$

where $p_{\nu}$ denotes the $\nu$-th prime number. This can be seen as a first effective version of Kronecker's approximation theorem, with a bound for $\tau$ (similar to the one in Dirichlet's approximation theorem). In view of (5) this yields, in addition with the easier case of bounding $|\zeta(s)|$ from below, the existence of infinite sequences $s_{ \pm}=\sigma_{ \pm}+i t_{ \pm}$with $\sigma_{ \pm} \rightarrow 1+$ and $t_{ \pm} \rightarrow+\infty$ for which

$$
\left|\zeta\left(s_{+}\right)\right| \geq A \log \log t_{+} \quad \text { and } \quad \frac{1}{\left|\zeta\left(s_{-}\right)\right|} \geq A \log \log t_{-},
$$

where $A>0$ is an absolute constant. However, for Dirichlet $L$-functions we need a more general effective version of Kronecker's approximation theorem. Using the idea of Bohr and Landau in addition with Baker's estimate for linear forms, Rieger [6] proved the remarkable

Theorem 2.1. Let $v, N \in \mathbb{N}, b \in \mathbb{Z}, 1 \leq \omega, U \in \mathbb{R}$. Let $p_{1}<\ldots<p_{N}$ be prime numbers (not necessarily consecutive) and

$$
u_{\nu} \in \mathbb{Z}, \quad 0<\left|u_{\nu}\right| \leq U, \quad \beta_{\nu} \in \mathbb{R} \quad \text { for } \quad \nu=1, \ldots, N .
$$

Then there exist $h_{\nu} \in \mathbb{Z}, 0 \leq \nu \leq N$, and an effectively computable number $C=C\left(N, p_{N}\right)>0$, depending on $N$ and $p_{N}$ only, with

$$
\left|h_{0} \frac{u_{\nu}}{v} \log p_{\nu}-\beta_{\nu}-h_{\nu}\right|<\frac{1}{\omega} \quad \text { for } \quad \nu=1, \ldots, N
$$

and $b \leq h_{0} \leq b+(2 U v \omega)^{C}$.

We need $C$ explicitly. Therefore we shall give a sketch of Rieger's proof and add in the crucial step a result on an explicit lower bound for linear forms in logarithms due to Waldschmidt [9].

Let $\mathbb{K}$ be a number field of degree $D$ over $\mathbb{Q}$ and denote by $L_{\mathbb{K}}$ the set of logarithms of the elements of $\mathbb{K} \backslash\{0\}$, i.e.,

$$
L_{\mathbb{K}}=\{\ell \in \mathbb{C}: \exp (\ell) \in \mathbb{K}\} .
$$

If $a$ is an algebraic number with minimal polynomial $P(X)$ over $\mathbb{Z}$, then define the absolute logarithmic height of $a$ by

$$
h(a)=\frac{1}{D} \int_{0}^{1} \log |P(\exp (2 \pi i \phi))| \mathrm{d} \phi ;
$$

note that $h(a)=\log a$ for integers $a \geq 2$. Waldschmidt proved 
Theorem 2.2. Let $\ell_{\nu} \in L_{\mathbb{K}}$ and $\beta_{\nu} \in \mathbb{Q}$ for $\nu=1, \ldots, N$, not all equal zero. Define $a_{\nu}=\exp \left(\ell_{\nu}\right)$ for $\nu=1, \ldots, N$ and

$$
\Lambda=\beta_{0}+\beta_{1} \log a_{1}+\ldots+\beta_{N} \log a_{N} .
$$

Let $E, W$ and $V_{\nu}, 1 \leq \nu \leq N$, be positive real numbers, satisfying

$$
\begin{gathered}
W \geq \max _{1 \leq \nu \leq N}\left\{h\left(\beta_{\nu}\right)\right\}, \\
\frac{1}{D} \leq V_{1} \leq \ldots \leq V_{N}, \\
V_{\nu} \geq \max \left\{h\left(a_{\nu}\right), \frac{\left|\log a_{\nu}\right|}{D}\right\} \quad \text { for } \quad \nu=1, \ldots, N
\end{gathered}
$$

and

$$
1<E \leq \min \left\{\exp \left(V_{1}\right), \min _{1 \leq \nu \leq N}\left\{\frac{4 D V_{\nu}}{\left|\log a_{\nu}\right|}\right\}\right\} .
$$

Finally, define $V_{\nu}^{+}=\max \left\{V_{\nu}, 1\right\}$ for $\nu=N$ and $\nu=N-1$, with $V_{1}^{+}=1$ in the case $N=1$. If $\Lambda \neq 0$, then

$$
\begin{aligned}
&|\Lambda|>\exp \left(-c(N) D^{N+2}\left(W+\log \left(E D V_{N}^{+}\right)\right) \log \left(E D V_{N-1}^{+}\right) \times\right. \\
&\left.\times(\log E)^{-N-1} \prod_{\nu=1}^{N} V_{\nu}\right)
\end{aligned}
$$

with $c(N) \leq 2^{8 N+51} N^{2 N}$.

This leads to

Theorem 2.3. With the notation of Theorem 2.1 and under its assumptions there exists an integer $h_{0}$ such that (11) holds and

$$
b \leq h_{0} \leq b+2+\left(\left(3 \omega U(N+2) \log p_{N}\right)^{4}+2\right)^{N+2} \times
$$

$$
\times \exp \left(2^{8 N+51} N^{2 N}\left(1+2 \log p_{N}\right)\left(1+\log p_{N-1}\right) \prod_{\nu=2}^{N} \log p_{\nu}\right) ;
$$

if $p_{N}$ is the $N$-th prime number, then, for any $\epsilon>0$ and $N$ sufficiently large,

$$
b \leq h_{0} \leq b+(\omega U)^{(4+\epsilon) N} \exp \left(N^{(2+\epsilon) N}\right) .
$$

Proof. For $t \in \mathbb{R}$ define

$$
f(t)=1+\exp (t)+\sum_{\nu=1}^{N} \exp \left(2 \pi i\left(t \frac{u_{\nu}}{v} \log p_{\nu}-\beta_{\nu}\right)\right) .
$$


With $\gamma_{-1}:=0, \beta_{-1}:=0, \gamma_{0}:=1, \beta_{0}:=0$ and $\gamma_{\nu}:=\frac{u_{\nu}}{v} \log p_{\nu}, 1 \leq \nu \leq N$, we have

$$
f(t)=\sum_{\nu=-1}^{N} \exp \left(2 \pi i\left(t \gamma_{\nu}-\beta_{\nu}\right)\right)
$$

By the multinomial theorem,

$$
f(t)^{k}=\sum_{\substack{j \nu \geq 0 \\ j-1+\cdots+j_{N}=k}} \frac{k !}{j_{-1} ! \cdots j_{N} !} \exp \left(2 \pi i \sum_{\nu=-1}^{N} j_{\nu}\left(t \gamma_{\nu}-\beta_{\nu}\right)\right) .
$$

Hence, for $0<B \in \mathbb{R}$ and $k \in \mathbb{N}$

$$
\begin{aligned}
J:= & \int_{b}^{b+B}|f(t)|^{2 k} \mathrm{~d} t \\
= & \sum_{\substack{j \nu \geq 0 \\
j_{-1}+\cdots+j_{N}=k}} \frac{k !}{j_{-1} ! \cdots j_{N} !} \sum_{\substack{j \nu^{\prime} \geq 0 \\
j_{-1}^{\prime}+\cdots+j_{N}^{\prime}=k}} \frac{k !}{j_{-1}^{\prime} ! \cdots j_{N}^{\prime} !} \\
& \int_{b}^{b+B} \exp \left(2 \pi i\left(\sum_{\nu=-1}^{N}\left(j_{\nu}-j_{\nu}^{\prime}\right) \gamma_{\nu} t-\sum_{\nu=-1}^{N}\left(j_{\nu}-j_{\nu}^{\prime}\right) \beta_{\nu}\right)\right) \mathrm{d} t .
\end{aligned}
$$

By the theorem of Lindemann

$$
\sum_{\nu=-1}^{N}\left(j_{\nu}-j_{\nu}^{\prime}\right) \gamma_{\nu}
$$

vanishes if and only if $j_{\nu}=j_{\nu}^{\prime}$ for $\nu=-1,0, \ldots, N$. Thus, integration gives

$$
\int_{b}^{b+B} \exp \left(2 \pi i\left(\sum_{\nu=-1}^{N}\left(j_{\nu}-j_{\nu}^{\prime}\right) \gamma_{\nu} t-\sum_{\nu=-1}^{N}\left(j_{\nu}-j_{\nu}^{\prime}\right) \beta_{\nu}\right)\right) \mathrm{d} t=B
$$

if $j_{\nu}=j_{\nu}^{\prime}, \nu=-1,0, \ldots, N$, and

$$
\begin{aligned}
\left|\int_{b}^{b+B} \exp \left(2 \pi i\left(\sum_{\nu=-1}^{N}\left(j_{\nu}-j_{\nu}^{\prime}\right) \gamma_{\nu} t-\sum_{\nu=-1}^{N}\left(j_{\nu}-j_{\nu}^{\prime}\right) \beta_{\nu}\right)\right) \mathrm{d} t\right| \\
\leq \frac{1}{\pi}\left|\sum_{\nu=-1}^{N}\left(j_{\nu}-j_{\nu}^{\prime}\right) \gamma_{\nu}\right|^{-1}
\end{aligned}
$$

if $j_{\nu} \neq j_{\nu}^{\prime}$ for some $\nu \in\{-1,0, \ldots, N\}$. In the latter case there exists by Baker's estimate for linear forms an effectively computable constant $A$ such that

$$
\left|\sum_{\nu=-1}^{N}\left(j_{\nu}-j_{\nu}^{\prime}\right) \gamma_{\nu}\right|^{-1}<A
$$


Setting $\beta_{0}=j_{0}-j_{0}^{\prime}, \beta_{\nu}=\frac{u_{\nu}}{v}\left(j_{\nu}-j_{\nu}^{\prime}\right)$ and $a_{\nu}=p_{\nu}$ for $\nu=1, \ldots, N$, we have, with the notation of Theorem 2.2 ,

$$
\Lambda=\sum_{\nu=-1}^{N}\left(j_{\nu}-j_{\nu}^{\prime}\right) \gamma_{\nu}
$$

We may take $E=1, W=\log p_{N}, V_{1}=1$ and $V_{\nu}=\log p_{\nu}$ for $\nu=2, \ldots, N$. If $N \geq 2$, Theorem 2.2 gives

$$
|\Lambda|>\exp \left(-2^{8 N+51} N^{2 N}\left(1+2 \log p_{N}\right)\left(1+\log p_{N-1}\right) \prod_{\nu=2}^{N} \log p_{\nu}\right) .
$$

Thus we may take

$$
A=\exp \left(2^{8 N+51} N^{2 N}\left(1+2 \log p_{N}\right)\left(1+\log p_{N-1}\right) \prod_{\nu=2}^{N} \log p_{\nu}\right) .
$$

Hence, we obtain

$$
\begin{aligned}
\text { (16) } J \geq B & \sum_{\substack{j \nu \geq 0 \\
j_{-1}+\cdots+j_{N}=k}}\left(\frac{k !}{j_{-1} ! \cdots j_{N} !}\right)^{2} \\
& -\frac{A}{\pi} \sum_{\substack{j \nu \geq 0 \\
j_{-1}+\cdots+j_{N}=k}} \frac{k !}{j_{-1} ! \cdots j_{N} !} \sum_{\substack{j \nu^{\prime} \geq 0 \\
j_{-1}^{\prime}+\cdots+j_{N}^{\prime}=k}} \frac{k !}{j_{-1}^{\prime} ! \cdots j_{N}^{\prime} !} .
\end{aligned}
$$

Since

$$
\sum_{\substack{j \nu \geq 0 \\ j-1+\ldots+j_{N}=k}} 1 \leq(k+1)^{N+2},
$$

application of the Cauchy Schwarz-inequality to the first multiple sum and of the multinomial theorem to the second multiple sum on the right hand side of (16) yields

$$
\begin{aligned}
J & \geq\left(\frac{B}{(k+1)^{N+2}}-\frac{A}{\pi}\right)\left(\sum_{\substack{j \nu \geq 0 \\
j-1 \\
j+\cdots+j_{N}=k}} \frac{k !}{j_{-1} ! \cdots j_{N} !}\right)^{2} \\
& \geq\left(\frac{B}{(k+1)^{N+2}}-\frac{A}{\pi}\right)(N+2)^{2 k} .
\end{aligned}
$$

Setting $B=A(k+1)^{N+2}$ and with $\tau \in[b, b+B]$ defined by

$$
|f(\tau)|=\max _{t \in[b, b+B]}|f(t)|,
$$

we obtain

$$
\frac{B(N+2)^{2 k}}{2(k+1)^{N+2}} \leq J \leq B|f(\tau)|^{2 k}
$$


This gives

$$
|f(\tau)|>N+2-2 \mu, \quad \text { where } \quad \mu:=\frac{(N+2)^{2} \log k}{3 k} ;
$$

note that $\mu<1$ for $k \geq 11$. By definition

$$
f(t)=1+\exp \left(2 \pi i\left(t \gamma_{\nu}-\beta_{\nu}\right)\right)+\sum_{\substack{m=0 \\ m \neq \nu}}^{N} \exp \left(2 \pi i\left(t \gamma_{m}-\beta_{m}\right)\right) .
$$

Therefore, using the triangle inequality,

$$
|f(t)| \leq N+\left|1+\exp \left(2 \pi i\left(\tau \gamma_{\nu}-\beta_{\nu}\right)\right)\right| \quad \text { for } \quad \nu=0, \ldots, N,
$$

and arbitrary $t \in \mathbb{R}$. Thus, in view of (17)

$$
\left|1+\exp \left(2 \pi i\left(\tau \gamma_{\nu}-\beta_{\nu}\right)\right)\right|>2-2 \mu \quad \text { for } \quad \nu=0, \ldots, N .
$$

If $h_{\nu}$ denotes the nearest integer to $\tau \gamma_{\nu}-\beta_{\nu}$, then

$$
\left|\tau \gamma_{\nu}-\beta_{\nu}-h_{\nu}\right|<\sqrt{\frac{\mu}{2}} \quad \text { for } \quad \nu=0, \ldots, N .
$$

For $\nu=0$ this implies $\left|\tau-h_{0}\right|<\sqrt{\mu}$. Replacing $\tau$ by $h_{0}$ yields

$$
\left|h_{0} \gamma_{\nu}-\beta_{\nu} h_{\nu}\right|<\sqrt{\mu}\left(1+\max _{\nu=1, \ldots, N}\left|\gamma_{\nu}\right|\right) \quad \text { for } \quad \nu=1, \ldots, N \text {. }
$$

Putting $k=\left[\left(3 w U(N+2) \log p_{N}\right)^{4}\right]+1$ we get

$$
b-1 \leq h_{0} \leq b+1+B=b+1+A\left(\left[\left(3 \omega U(N+2) \log p_{N}\right)^{4}\right]+2\right)^{N+2} .
$$

Substituting (15) and replacing $b-1$ by $b$, the assertion of Theorem 2.1 follows with the estimate (12) of Theorem $2.3 ;(13)$ can be proved by standard estimates.

\section{Quantitative results}

We continue with inequality (9). Let $p_{N}$ be the $N$-th prime. Then, using Theorem 2.3 with $N=\pi(x), v=u_{\nu}=1$, and

$$
\beta_{\nu}=\frac{\lambda_{p_{\nu}}}{\varphi(q)}+\frac{\theta}{2 \pi} \quad \text { for } \quad \nu=1, \ldots, N
$$

yields the existence of $\tau=2 \pi h_{0}$ with

$$
b \leq \frac{\tau}{2 \pi} \leq b+\omega^{(4+\epsilon) N} \exp \left(N^{(2+\epsilon) N}\right)
$$

such that (4) holds, as $N$ and $x$ tend to infinity. We choose $\omega=\log \log x$, then the prime number theorem and (18) imply

$$
\log x=\log N+O(\log \log N), \quad \log N \geq \log \log \log \tau+O(\log \log \log \log \tau) .
$$

Substituting this in (9) we obtain 
Theorem 3.1. For any real $\theta$ there are infinitely many values of $s=\sigma+i t$ with $\sigma \rightarrow 1+$ and $t \rightarrow+\infty$ such that

$$
R e\{\exp (i \theta) \log L(s, \chi)\} \geq \log \frac{\log \log \log t}{\log \log \log \log t}+O(1) .
$$

Using the Phragmén-Lindelöf principle, it is even possible to get quantitative estimates on the abscissa of absolute convergence. We write $f(x)=$ $\Omega(g(x))$ with a positive function $g(x)$ if

$$
\liminf _{x \rightarrow \infty} \frac{|f(x)|}{g(x)}>0
$$

hence, $f(x)=\Omega(g(x))$ is the negation of $f(x)=o(g(x))$. Then, by the same reasoning as in $[8], \S 8.4$, we deduce

$$
L(1+i t, \chi)=\Omega\left(\frac{\log \log \log t}{\log \log \log \log t}\right),
$$

and

$$
\frac{1}{L(1+i t, \chi)}=\Omega\left(\frac{\log \log \log t}{\log \log \log \log t}\right) .
$$

However, the method of Ramachandra [5] yields better results. As for the Riemann zeta-function (10) it can be shown that

$$
L(1+i t, \chi)=\Omega(\log \log t), \quad \text { and } \quad \frac{1}{L(1+i t, \chi)}=\Omega(\log \log t),
$$

and further that, assuming Riemann's hypothesis, this is the right order (similar to [8], §14.8). Hence, it is natural to expect that also in the halfplane of absolute convergence for Dirichlet $L$-functions similar growth estimates as for the Riemann zeta-function (10) should hold. We give a heuristical argument. Weyl improved Kronecker's approximation theorem by

Theorem 3.2. Let $a_{1}, \ldots, a_{N} \in \mathbb{R}$ be linearly independent over the field of rational numbers, and let $\gamma$ be a subregion of the $N$-dimensional unit cube with Jordan volume $\Gamma$. Then

$$
\lim _{T \rightarrow \infty} \frac{1}{T} \operatorname{meas}\left\{\tau \in(0, T):\left(a_{1} t, \ldots, a_{N} t\right) \in \gamma \bmod 1\right\}=\Gamma .
$$

Since the limit does not depend on translations of the set $\gamma$, we do not expect any deep influence of the inhomogeneous part to our approximation problem (4) (though it is a question of the speed of convergence). Thus, we may conjecture that we can find a suitable $\tau \leq \exp \left(N^{c}\right)$ with some positive constant $c$ instead of (13), as in Dirichlet's homogeneous approximation theorem. This would lead to estimates similar to (10). 
We conclude with some observations on the density of extremal values of $\log L(s, \chi)$. First of all note that if

$$
|L(1+i \tau, \chi)|^{ \pm 1} \geq f(T)
$$

holds for a subset of values $\tau \in[T, 2 T]$ of measure $\mu T$, where $f(T)$ is any function which tends with $T$ to infinity, then

$$
\int_{T}^{2 T}|L(1+i t, \chi)|^{ \pm 2} \mathrm{~d} t \geq \mu T f(T)^{2} .
$$

In view of well-known mean-value formulae we have $\mu=0$, which implies

$$
\lim _{T \rightarrow \infty} \frac{1}{T} \operatorname{meas}\left\{\tau \in[0, T]:|L(\sigma+i \tau)|^{ \pm 1} \geq f(T)\right\}=0 .
$$

This shows that the set on which extremal values are taken is rather thin.

The situation is different for fixed $\sigma>1$. Let $Q$ be the smallest prime $p$ for which $\chi_{0}(p) \neq 0$. Then

$$
|\log L(s, \chi)| \leq \log L\left(\sigma, \chi_{0}\right)=Q^{-\sigma}\left(1+O\left(\left(\frac{Q}{Q+1}\right)^{\sigma}\right)\right) ;
$$

note that the right hand side tends to $0+$ as $\sigma \rightarrow+\infty$, and that $Q \leq q+1$.

Theorem 3.3. Let $0<\delta<\frac{1}{2}$. Then, for arbitrary $\theta$ and fixed $\sigma>1$,

$$
\begin{gathered}
\liminf _{M \rightarrow \infty} \frac{1}{M} \sharp\left\{m \leq M:(1-\delta) \log L\left(\sigma, \chi_{0}\right)-\operatorname{Re}\{\exp (i \theta) \log L(\sigma+2 \pi i m, \chi)\}\right\} \\
\geq Q^{-2 \sigma}\left(1+\frac{24}{\sigma}\right) \geq \delta^{2 Q^{2}+8}(2 Q)^{-8 Q^{2}-32} \exp \left(-2^{3 Q^{2}+51} Q^{4 Q^{2}+2}\right) .
\end{gathered}
$$

Proof. We omit the details. First, we may replace (2) by

$$
\left|\log L(s, \chi)-\sum_{p} \frac{\chi(p)}{p^{s}}\right| \leq \sum_{p, k \geq 2} \frac{\chi_{0}(p)}{k p^{k \sigma}} .
$$

This gives with regard to (8)

$\operatorname{Re}\{\exp (i \theta) \log L(\sigma+2 \pi i m, \chi)\} \geq(1-\delta) \log L\left(\sigma, \chi_{0}\right)-2 \frac{x^{1-\sigma}}{\sigma-1}-8 \frac{Q^{2-2 \sigma}}{2^{\sigma}(\sigma-1)}$ for some integer $h_{0}=m$, satisfying (12), where $N=\pi(x)$ and $\cos \frac{2 \pi}{\omega}=1-\delta$. Putting $x=Q^{2}$, proves (after some simple computation) the theorem.

For example, if $\chi$ is a character with odd modulus $q$, then the quantity of Theorem 3.3 is bounded below by

$$
\geq \frac{\delta^{16}}{2^{128} \exp \left(2^{81}\right)} .
$$


Acknowledgement. The author would like to thank Prof. A. Laurinčikas, Prof. G.J. Rieger and Prof. W. Schwarz for their constant interest and encouragement.

\section{References}

[1] H. Bohr, E. LANDAU, Über das Verhalten von $\zeta(s)$ und $\zeta^{(k)}(s)$ in der Nähe der Geraden $\sigma=1$. Nachr. Ges. Wiss. Göttingen Math. Phys. Kl. (1910), 303-330.

[2] H. Bohr, E. Landau, Nachtrag zu unseren Abhandlungen aus den Jahren 1910 und 1923. Nachr. Ges. Wiss. Göttingen Math. Phys. Kl. (1924), 168-172.

[3] H. Davenport, H. Heilbronn, On the zeros of certain Dirichlet series I, II. J. London Math. Soc. 11 (1936), 181-185, 307-312.

[4] R. Garunkštis, On zeros of the Lerch zeta-function II. Probability Theory and Mathematical Statistics: Proceedings of the Seventh Vilnius Conf. (1998), B.Grigelionis et al. (Eds.), TEV/Vilnius, VSP/Utrecht, 1999, 267-276.

[5] K. Ramachandra, On the frequency of Titchmarsh's phenomenon for $\zeta(s)-V I I$. Ann. Acad. Sci. Fennicae 14 (1989), 27-40.

[6] G.J. RIEGER, Effective simultaneous approximation of complex numbers by conjugate algebraic integers. Acta Arith. 63 (1993), 325-334.

[7] E.C. Titchmarsh, The theory of functions. Oxford University Press, 1939 2nd ed.

[8] E.C. Titchmarsh, The theory of the Riemann zeta-function. Oxford University Press, 1986 2nd ed.

[9] M. Waldschmidt, A lower bound for linear forms in logarithms. Acta Arith. 37 (1980), 257-283.

[10] H. WeYL, Über ein Problem aus dem Gebiete der diophantischen Approximation. Göttinger Nachrichten (1914), 234-244

Jörn STEUding

Institut für Algebra und Geometrie

Fachbereich Mathematik

Johann Wolfgang Goethe-Universität Frankfurt

Robert-Mayer-Str. 10

60054 Frankfurt, Germany

E-mail : steuding@math.uni-frankfurt.de 